\title{
The birth environment of the solar system constrained by the relative abundances of the solar radionuclides
}

\author{
Edward D. Young ${ }^{1}$ \\ ${ }^{1}$ Department of Earth, Planetary, and Space Sciences, \\ University of California, Los Angeles \\ email: eyoung@epss.ucla.edu
}

\begin{abstract}
The relative abundances of the radionuclides in the solar system at the time of its birth are crucial arbiters for competing hypotheses regarding the birth environment of the Sun. The presence of short-lived radionuclides, as evidenced by their decay products in meteorites, has been used to suggest that particular, sometimes exotic, stellar sources were proximal to the Sun's birth environment. The recent confirmation of neutron star - neutron star (NS-NS) mergers and associated kilonovae as potentially dominant sources of r-process nuclides can be tested in the case of the solar birth environment using the relative abundances of the longer-lived nuclides. Critical analysis of the 15 radionuclides and their stable partners for which abundances and production ratios are well known suggests that the Sun formed in a typical massive starforming region (SFR). The apparent overabundances of short-lived radionuclides (e.g. ${ }^{26} \mathrm{Al}$, ${ }^{41} \mathrm{Ca},{ }^{36} \mathrm{Cl}$ ) in the early solar system appears to be an artifact of a heretofore under-appreciation for the important influences of enrichment by Wolf-Rayet winds in SFRs. The long-lived nuclides (e.g. ${ }^{238} \mathrm{U},{ }^{244} \mathrm{Pu},{ }^{247} \mathrm{Cr},{ }^{129} \mathrm{I}$ ) are consistent with an average time interval between production events of $10^{8}$ years, seemingly too short to be the products of NS-NS mergers alone. The relative abundances of all of these nuclides can be explained by their mean decay lifetimes and an average residence time in the ISM of $\sim 200$ Myr. This residence time evidenced by the radionuclides is consistent with the average lifetime of dust in the ISM and the timescale for converting molecular cloud mass to stars.
\end{abstract}

Keywords. ISM, stars: Wolf-Rayet, solar system: formation, Sun: abundances

\section{Introduction}

A worthy goal for studying the origins of the solar system is to establish whether or not the Sun formed in a typical environment and under typical conditions as judged by comparisons with star-forming regions in the Milky Way today. Advantages of making this link between the birth of our solar system and that of other planetary systems in general are twofold. Firstly, a reconstruction of the birth environment of the Sun is relevant to assessing the probability that planetary systems analogous to our solar system exist, have existed, or will exist in the Milky Way Galaxy. Secondly, if we establish the basic characteristics of the solar birth environment, we can test our understanding of star and planet formation in analogous environments using the solar system as the most well understood example.

Among the many physical and chemical characteristics of the solar system, the relative abundances of the radionuclides may be some of the best clues to the solar birth environment. The initial relative abundances of many radionuclides in the solar system have been obtained through painstaking measurements of the isotopes themselves in meteorites, or, in the case of the short-lived radionuclides (SLRs), their decay products in 
meteorites. Here we present two different paradigms for interpreting the meaning of the relative abundances of the radionuclides and their implications for reconstructing the environment in which our solar system formed. The two views are in effect basis vectors for all models seeking to explain the solar abundances of the radionuclides. The argument is made that initial abundances of SLRs are likely to be typical of massive star-forming regions (SFRs) similar to the Cygnus or Carina regions in our Galaxy today.

\section{Overview}

Theories for the provenance of the solar-system radionuclides can be divided into two broad categories. In the first category, which we will refer to here as "punctuated delivery", the radiochemistry of the solar system is the result of the mingling of materials from a variety of discrete nucleosynthesis sources that are sometimes attributed to individual stars of specified masses and ages, including AGB stars, supernovae (SNe), and Wolf-Rayet stars (e.g., Wasserburg et al. 1996, 2006; Lugaro et al. 2014, Dwarkadas et al. 2017). The punctuated delivery scenarios emphasize the "granularity" (Wasserburg et al. 1996) of the interstellar medium in that a chance encounter with a single stellar source can dominate the inventory of a particular nuclide comprising pre-stellar material. In this type of interpretation, the solar abundances of radionuclides are the result of a sequence of random discrete events (e.g., the explosion of a proximal supernova).

The second category of explanations for the solar abundances of the radionuclides appeals to widespread, quasi-continuous self-enrichment of massive SFRs (Jura et al. 2013; Young 2014, Fujimoto et al. 2018). In these scenarios, abundances of SLRs represent a steady-state resulting from quasi-continuous production and losses in the SFRs over time. The chemistry of the solar system therefore reflects the global evolution of the SFR in which the Sun formed. Some models are of course a mix of the two categories (e.g., Gounelle \& Meynet 2012), representing vectors in the two-dimensional cartesian space spanned by the two theories described here. In what follows the essence of the two distinct types of scenarios are compared using simple mathematical formulations. The results are displayed in plots of the relative abundances of radionuclides normalized for differences in chemistry and differences in stellar production rates against their average lifetimes imposed by radioactive decay. An eventual assessment of which of these two distinct types of scenarios is most likely will place constraints on the events leading up to the formation of the solar system, and thus the solar birth environment.

\section{Punctuated Delivery}

The punctuated nature, or "granularity" (Wasserburg et al. 1996), of stellar nucleosynthesis events that could have seeded parental solar system material with nuclides can be described by an equation based on a geometric series summing individual nucleosynthesis events with an average temporal spacing $\delta t$ followed by a final actual free decay time $\Delta t$ (Wasserburg et al. 2006, Lugaro et al. 2014)

$$
\frac{N_{\mathrm{R}}}{N_{\mathrm{S}}}=\left[\frac{P_{\mathrm{R}}}{P_{\mathrm{S}}} \frac{\delta t}{T}\left(1+\frac{\mathrm{e}^{-\delta \mathrm{t} / \tau}}{1-\mathrm{e}^{-\delta \mathrm{t} / \tau}}\right)\right] \exp (-\Delta t / \tau)
$$

where $N_{i}$ and $P_{i}$ are the number and production rates for radionuclides $(\mathrm{R})$ and stable isotope partner $(\mathrm{S})$, respectively, $T$ is the age of the Galaxy (taken to be 7.4 Gyr at the time of solar system formation), $\Delta t$ is in this case the time interval between the last event 
(LE) and the birth of the solar system, and $\tau$ is the mean life of $\mathrm{R}$ against radioactive decay. Dividing $N_{\mathrm{R}}$ by $N_{\mathrm{S}}$ eliminates the effects of element-specific chemistry when evaluating Equation 3.1 for different radionuclides. $N_{\mathrm{R}} / N_{\mathrm{S}}$ ratios are well known for the early solar system from precise measurements of the radioactive decay products of the SLRs and the relative abundances of the longer-lived nuclides in meteoritical materials (Huss et al. 2009). Comparisons of different radionuclides are further facilitated by dividing the abundance ratio on the left-hand side of Equation 3.1 by the production ratio on the right-hand side of Equation 3.1. Production ratios are obtained from models of stellar nucleosynthesis (e.g., Rauscher et al. 2002, Woosley \& Heger 2007; Sukhbold et al. 2018). The resulting quotient, $\alpha(R)=\left(N_{\mathrm{R}} / N_{\mathrm{S}}\right) /\left(P_{\mathrm{R}} / P_{\mathrm{S}}\right)$, for different radionuclides depends on the radioactive mean lifetime $\tau$ and the duration of radioactive decay between events, all else equal. Greater time intervals of decay following nucleosynthesis produce steeper trajectories in plots of $\alpha(R)$ vs $\tau$. If the radionuclides inherited by the solar system from its parent molecular cloud were all of similar average age, $\alpha(R)$ should vary only with $\tau$, with the shorter-lived nuclides being much less abundant relative to their production rates than the longer-lived nuclides.

Young (2016) showed that Equation 3.1 can be used to group the solar radionuclides for which production ratios are well known. Figure 1 shows updated curves of $\log \left[\left(N_{\mathrm{R}} / N_{\mathrm{S}}\right) /\left(P_{\mathrm{R}} / P_{\mathrm{S}}\right)\right]$, or equivalently $\log (\alpha(R))$, versus $\log (\tau)$, defining distinct groups of radionuclides. These groupings succinctly summarize many of the relationships previously described in the literature (e.g., Wasserburg et al. 2006, Lugaro et al. 2014). For example, the relative concentrations of r-process neutron-capture products ${ }^{129} \mathrm{I},{ }^{244} \mathrm{Pu}$, ${ }^{247} \mathrm{Cm},{ }^{235} \mathrm{U}$ and ${ }^{238} \mathrm{U}$ and the p-process nuclide ${ }^{146} \mathrm{Sm}$ are all explained by Equation (1) using $\delta t=10 \mathrm{Myr}$ and $\Delta t=100 \mathrm{Myr}$ (Figure 1, where ${ }^{232} \mathrm{Th}$ is used as the nearly stable nuclide to calculate $\alpha(R)$ for the actinides). Here we label these isotopes as Group I. The value for $\delta t$ is consistent with the frequency of core-collapse supernova (CCSN) events affecting random positions in the Galactic disk (Meyer \& Clayton 2000) and so is appropriate if r-process nuclides form mainly in CCSNe. Note that $\delta t$ is not the frequency of supernovae anywhere in the Galaxy, which is of order 1 per $50 \mathrm{yrs}$, but rather the frequency with which a random position in the Galaxy experiences a supernova explosion.

However, mounting evidence suggests that a principal source of r-process nuclides may be kilonovae resulting from mergers of neutron stars (NS-NS mergers) and possibly NSblack hole (NS-BH) mergers (Thielemann et al. 2017; Kasen et al. 2017; Smartt et al. 2017). Assuming similar production ratios for both CCSNe and NS-NS mergers (but see Cote et al. 2018), especially among the actinides, Figure 1 provides a constraint on the frequency of NS-NS mergers under the assumption that they were the primary source of solar r-process nuclides. For example, Figure 1 shows two curves where the frequency of nucleosynthesis events is either $100 \mathrm{Myr}$ or $1 \mathrm{Gyr}$ (for simplicity in this case we set $\Delta t=\delta t)$. The latter $\delta t$ of $1 \mathrm{Gyr}$ is in keeping with some estimates that NS-NS mergers are less frequent than CCSNe by a factor of 100 to 1000 (Cote et al. 2017, Tsujimoto \& Shigeyama 2014). The shorter event frequency $\delta t$ of 100 Myr fits the data reasonably well while the longer $\delta t$ of 1 Gyr does not fit the data. The implication is that either kilonovae events were only about ten times less frequent than CCSNe prior to solar system formation, or they were not the primary source of r-process nuclides leading up to the formation the Sun.

Lugaro et al. (2014) showed that a single set of values for $\delta t$ and $\Delta t$ can explain the relative concentrations of both s-process neutron addition nuclides ${ }^{107} \mathrm{Pd}$ and ${ }^{182} \mathrm{Hf}$. A value for $\delta t$ of $50 \mathrm{Myr}$, appropriate for AGB star encounters, and a similar value for $\Delta t$ of $40.35 \mathrm{Myr}$, fits the $\log \left[\left(N_{\mathrm{R}} / N_{\mathrm{S}}\right) /\left(P_{\mathrm{R}} / P_{\mathrm{S}}\right)\right]$ values for ${ }^{107} \mathrm{Pd}$ and ${ }^{182} \mathrm{Hf}$ (Figure 1 ). We refer to these radionuclides as Group II. ${ }^{53} \mathrm{Mn}$ and ${ }^{60} \mathrm{Fe}$ are treated separately from 


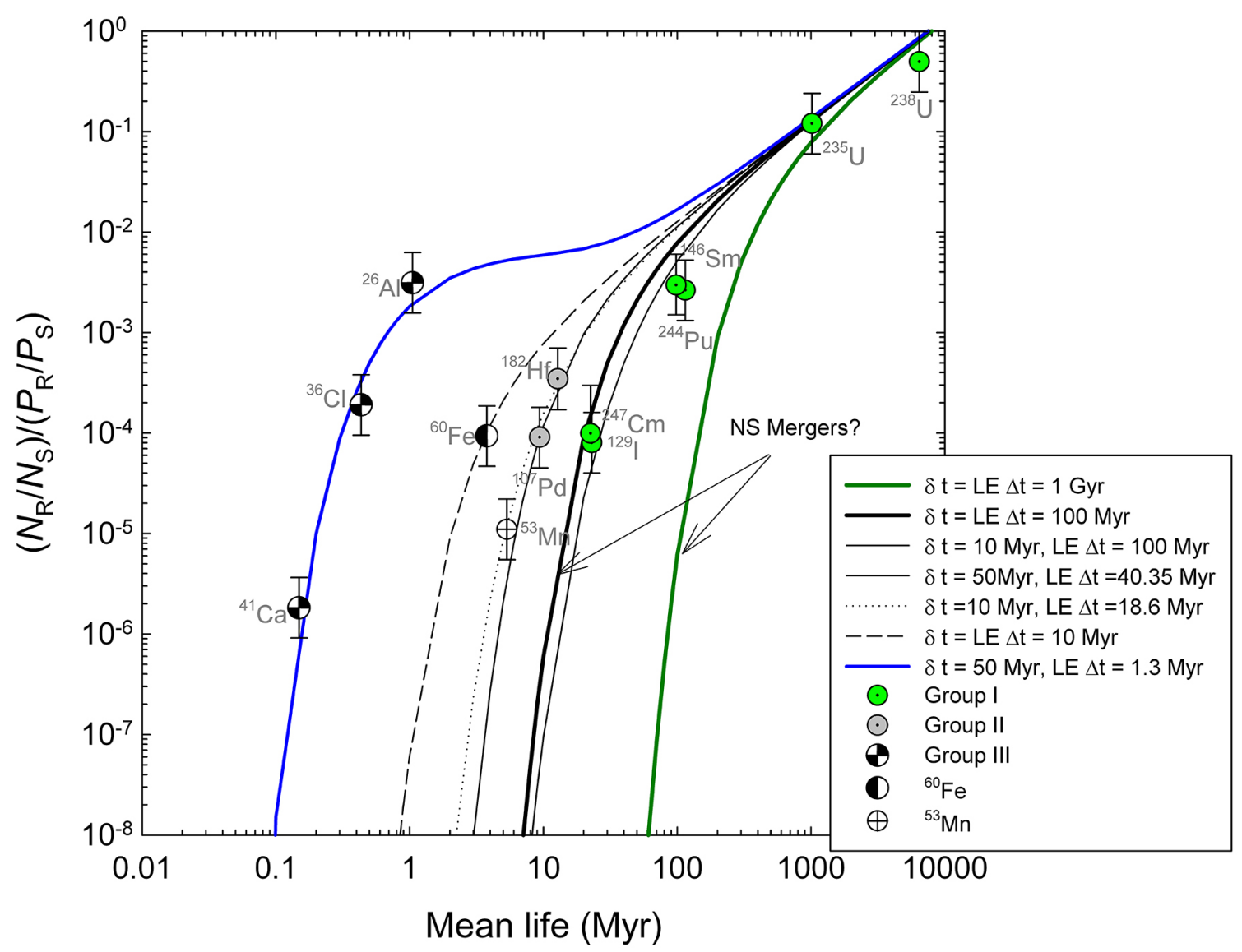

Figure 1. Plot of radionuclide abundances ratioed to their stable nuclide partners $\left(N_{\mathrm{R}} / N_{\mathrm{S}}\right)$ and production ratios $\left(P_{\mathrm{R}} / P_{\mathrm{S}}\right)$ (or $\alpha(R)$ as described in the text) vs. mean decay lifetimes for radionuclides present at the birth of the solar system. The values for the abundance ratios, production ratios, and decay lifetimes are from Young (2014) and references therein updated with new production ratios for ${ }^{182} \mathrm{Hf}$ and ${ }^{107} \mathrm{Pd}$ (Lugaro et al. 2014) and the ${ }^{247} \mathrm{Cm} /{ }^{232} \mathrm{Th}$ ratio and production ratio from Tissot et al. (2016) and Goriely \& Arnould (2001), respectively. Model curves are from Equation 3.1 fit to the different nuclide groups as indicated in the legend and in the text.

Group I and II. ${ }^{53} \mathrm{Mn}$ is also fit by the Group II curve but its origin must be distinct as it is a $\mathrm{SN}$ product, suggesting a shorter $\delta t$ interval. ${ }^{60} \mathrm{Fe}$ requires its own $\Delta t$ (Figure 1 ). The shortest-lived nuclides, labeled Group III, have $\log \left[\left(N_{\mathrm{R}} / N_{\mathrm{S}}\right) /\left(P_{\mathrm{R}} / P_{\mathrm{S}}\right)\right]$ values that are explained with Equation 3.1 using $\delta t=50 \mathrm{Myr}$ and $\Delta t=1.3 \mathrm{Myr}$ (Figure 1). The latter model reflects the fact that these short-lived nuclides have no "memory" of events prior to their most recent synthesis. Five distinct models defined by five $\Delta t$ values $(\delta t$ values are prescribed a priori by astrophysical constraints) represented by five curves are therefore required to explain the radionuclide abundances in Figure 1, one each for Groups I, II, and III and two others for ${ }^{53} \mathrm{Mn}$ and ${ }^{60} \mathrm{Fe}$.

In general, quantitative assessments of punctuated additions of SLRs to regions of star formation lead to relatively low probabilities for the initial solar abundances of SLRs 


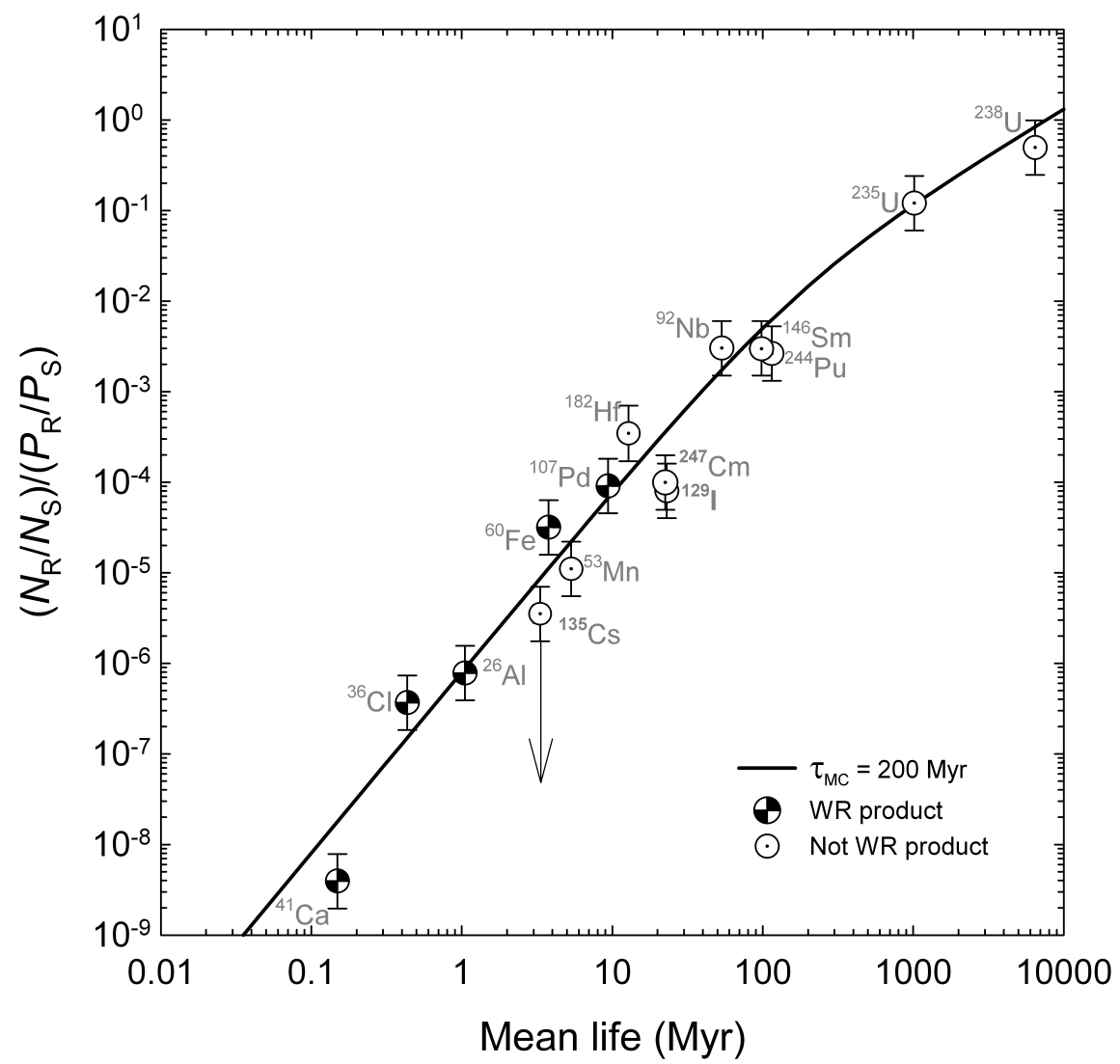

Figure 2. Plot of radionuclide abundances normalized to their stable nuclide partners $\left(N_{\mathrm{R}} / N_{\mathrm{S}}\right)$ and production ratios $\left(P_{\mathrm{R}} / P_{\mathrm{S}}\right)$ vs. mean decay lifetime for radionuclides in the early solar system. The plot is similar to Figure 1 but with ordinate values calculated using production ratios that include $\Lambda_{\mathrm{W}} / \Lambda_{\mathrm{SNe}}$ from Equation 4.2 and published WR production terms compiled by Young (2014). The curve is from Equation 4.1 fit to the data using $\Lambda_{\mathrm{W}} / \Lambda_{\mathrm{SNe}}=4000$ and $\tau_{\mathrm{MC}}=200$ Myr with a reduced $\chi^{2}$ of 0.95 . The value for ${ }^{92} \mathrm{Nb}$ and the upper limit for ${ }^{135} \mathrm{Cs}$ were not used to derive the best-fit curve, as discussed in the text.

(Adams 2010: Adams et al. 2014). In these scenarios, the solar system is assessed to be atypical in its complement of ${ }^{26} \mathrm{Al}$, for example.

\section{Quasi-continuous Self-enrichment of Star Forming Regions}

The quasi-continuous self-enrichment model for the solar birth environment relies on the enhanced production of several short-lived radionuclides emanating from Wolf-Rayet (WR) stars to explain the solar abundances of ${ }^{26} \mathrm{Al},{ }^{36} \mathrm{Cl}$, and ${ }^{41} \mathrm{Ca}$ at the time the solar system formed (referred to as the initial abundances). WR stars evolve from massive progenitors $\left(M_{*}>\sim 25 M_{\odot}\right)$. Their large progenitor masses ensure that the WR stage occurs within several Myr of the birth of the star cluster. WR stars are therefore expected to be spatially correlated with star-forming regions since they don't have time to flee their birth environments before they die (Young 2014). Young (2014) showed that the solar radionuclide abundances considered in Figure 1 are explained by a single model based 
on a two-phase interstellar medium (ISM) composed of a molecular cloud phase (MC) and a diffuse phase and with a molecular cloud mass fraction $x_{\mathrm{MC}}$ of $\sim 0.17$, equivalent to that today. The model equation (Young 2014, Jacobsen 2005) is

$$
\log \left(\frac{N_{\mathrm{R}, \mathrm{MC}}}{N_{\mathrm{S}, \mathrm{MC}}}\right)-\log \left(\frac{P_{\mathrm{R}}}{P_{\mathrm{S}}}\right)=2 \log \tau-\log \left[\left(1-x_{\mathrm{MC}}\right) \tau_{\mathrm{MC}}+\tau\right]-\log T
$$

where the abundances of radionuclides and their stable partners now refer to those in the molecular cloud phase and environs in the $\operatorname{SFR}\left(N_{\mathrm{R}, \mathrm{MC}}\right.$ and $N_{\mathrm{S}, \mathrm{MC}}$, respectively) as opposed to the diffuse ISM outside of the SFR. The radionuclide production terms relevant to the molecular cloud setting are cast in terms of production from supernovae $\left(P_{\mathrm{R}}^{\mathrm{SNe}}\right)$ and production from WR winds $\left(P_{\mathrm{R}}^{\mathrm{W}}\right)$ :

$$
\frac{P_{\mathrm{R}}}{P_{\mathrm{S}}}=\frac{\Lambda_{\mathrm{SNe}} P_{\mathrm{R}}^{\mathrm{SNe}}}{\Lambda_{\mathrm{SNe}} P_{\mathrm{S}}}+\frac{\Lambda_{\mathrm{W}} P_{\mathrm{R}}^{\mathrm{W}}}{\Lambda_{\mathrm{SNe}} P_{\mathrm{S}}}
$$

where $\Lambda_{\mathrm{W}}$ and $\Lambda_{\mathrm{SNe}}$ are the relative efficiencies for trapping the two sources of nuclides in the star-forming region. WR production values are listed by Young (2014).

Young (2016) showed that the model fits solar-system data using two independent parameters, an enhancement of WR wind production over SN production in SFRs, with $\Lambda_{\mathrm{W}} / \Lambda_{\mathrm{SNe}} \sim 4000$, and a sequestration time of nuclides in molecular cloud dust, $\tau_{\mathrm{MC}}$, of $\sim 200 \mathrm{Myr}$ (Figure 2). The $\tau_{\mathrm{MC}}$ in Equation 4.1 is the average time spent in SFR molecular clouds where they experience radioactive decay with no new additions before incorporation into stars. Because individual clouds exist for shorter time spans than the SFR as a whole, time spent passing from one cloud to another via inter-cloud space in the SFR is included in the residence time. The radionuclide residence time $\tau_{\mathrm{MC}}$ of $\sim 200$ Myr is consistent with the lifetime of dust in the interstellar medium (Tielens 2005) and simple estimates for the timescale for converting molecular clouds to stars (Draine 2011). In general, large values for $\Lambda_{\mathrm{W}} / \Lambda_{\mathrm{SNe}}$ are consistent with the fact that massive stars like WR progenitors apparently do not typically end their lives as energetic SNe but rather collapse by fallback to form black holes directly (Fryer 1999, Smartt 2009). In part for this reason, the products of WR winds are enhanced relative to SNe products in SFRs sufficiently massive as to host a population of large stars (Young 2014). The relatively quiescent ultimate fate of WR stars is supported by calculations showing that the fraction of stars with $M_{*}>18 M_{\odot}$ that explode rather than undergoing direct collapse is just $8 \%$ (Sukhbold et al. 2018).

Numerical simulations (e.g., Young 2014) show that if clusters form every 1 to $1.5 \mathrm{Myr}$ in an SFR, and if mixing is sufficiently efficient, then the abundances of ${ }^{26} \mathrm{Al},{ }^{36} \mathrm{Cl}$, and ${ }^{41} \mathrm{Ca}$ will be enhanced in SFRs relative to the average ISM as a whole. Jura et al. (2013) appealed to this concept when they pointed out that the initial solar relative abundance of ${ }^{26} \mathrm{Al}$, usually expressed as ${ }^{26} \mathrm{Al} /{ }^{27} \mathrm{Al}=5 \times 10^{-5}$, is indistinguishable from that deduced by assuming that the 1.5 to $2.5 M_{\odot}$ of ${ }^{26} \mathrm{Al}$ in the Galaxy (Martin et al. 2009) resides primarily in regions where stars are forming. These SFRs are represented to first order by the $8.4 \times 10^{8} M_{\odot}$ of $\mathrm{H}_{2}$ gas in the Galaxy (Draine 2011) and solar-like ratios of heavy elements. A correlation between SFRs and ${ }^{26} \mathrm{Al}$ in the Galaxy is supported by maps of the $1.8 \mathrm{MeV}$ gamma ray emission from ${ }^{26} \mathrm{Al}$ decay in the Galaxy (Diehl et al. 2006). The conclusion from this analysis is that the solar initial abundance of ${ }^{26} \mathrm{Al}$, and by inference those of ${ }^{36} \mathrm{Cl}$ and ${ }^{41} \mathrm{Ca}$ as well, was not extraordinary, but rather typical of star-forming regions as the result of self enrichment by WR stars. A recent numerical simulation at the Galactic scale by Fujimoto et al. (2018) leads to a similar conclusion regarding the 
importance of widespread self enrichment of SLRs in and near giant molecular clouds in the Galaxy.

The earlier work showing that a single curve in Figure 2 explains the solar abundances of radionuclides makes the tacit prediction that as more radionuclide initial abundances are obtained from measurements in meteorites, they too should fall on the same curve defined by the two parameters $\Lambda_{\mathrm{W}} / \Lambda_{\mathrm{SNe}} \sim 4000$ and $\tau_{\mathrm{MC}} \sim 200 \mathrm{Myr}$. This is because these two parameters should be intrinsic properties of the SFR in which the solar system formed. This prediction is put to the test by new data for the initial relative abundances of two additional short-lived radionuclides. These new data include an estimate for the initial abundance of the p-process product ${ }^{92} \mathrm{Nb}$, yielding ${ }^{92} \mathrm{Nb} /{ }^{93} \mathrm{Nb}=1.7 \pm 0.6 \times 10^{-5}$ (Iizuka et al. 2016), and a new upper limit on the initial abundance of the s-process product ${ }^{135} \mathrm{Cs}$, yielding ${ }^{135} \mathrm{Cs} /{ }^{133} \mathrm{Cs} \leqslant 2.8 \times 10^{-6}$ (Brennecka \& Kleine 2017). Using the mean production ratio for ${ }^{92} \mathrm{Nb} /{ }^{93} \mathrm{~N}$ of $5.65 \times 10^{-3}$ from Schonbachler et al. (2002) and the ${ }^{135} \mathrm{Cs} /{ }^{133} \mathrm{Cs}$ production ratio of 0.8 from Harper (1996), the new data plot within error of the solar-system curve in Figure 2. The new data are thus supportive of the prediction that all radionuclides, excluding those produced by spallation, will plot on the curve defined by $\Lambda_{\mathrm{W}} / \Lambda_{\mathrm{SNe}} \sim 4000$ and $\tau_{\mathrm{MC}} \sim 200 \mathrm{Myr}$. If this proves to be true as more data are acquired, we will have learned about two fundamental parameters that characterized the SFR in which the solar system formed.

\section{Model Selection and Concluding Remarks}

The two different interpretations of the solar-system radionuclide data portrayed in Figures 1 and 2 each have elements to recommend them. For example, the physical and temporal separation between r-process nucleosynthesis and production of lower-mass nuclides by CCSNe implied by the new kilonova data might be expressed by the different curves for these nuclides in Figure 1. Conversely, all 15 radionuclides for which we have good estimates of $\alpha(R)$ values can be fit with a single curve with 2 fit parameters in Figure 2 with an acceptable reduced $\chi^{2}$ of near unity. Parsimony would seem to be on the side of the quasi-continuous self-enrichment model. This point has been made using a simple Bayesian analysis (Young 2016). However, parsimony may not be the best arbiter for choosing between the two models described here. Nonetheless, if the self-enrichment model continues to explain more data coming from detailed analyses of meteorite samples, the physical and chemical characteristics of the solar system may prove useful for understanding better the nature of massive star-forming regions in general.

\section{References}

Adams, F. C. 2010, Annu. Rev. Astron. Astrophys., 48, 47

Adams, F. C., Fatuzzo, M., \& Holden, L. 2014, ApJ, 789, 18pp

Brennecka, G. A. \& Kleine, T. 2017, ApJL, 837, 6pp

Cote, B., Belczynski, K., Fryer, C. L., Ritter, C., Paul, A., Wehmeyer, B., \& O'Shea, B. W. 2017, ApJ, 836, 20pp

Cote, B., Eichler, M., Arcones, A., Hansen, C. J., Simonetti, P., Frebel, A., Fryer, C. L., Pignatari, M., Reichert, M., Belczynski, K., \& Matteucci, F. 2018, arXiv:1809.03525v2

Diehl, R., Halloin, H., Kretschmer, K., Lichti, G., Schonfelder, V., Strong, A., von Kienlin, A., Wang, W., Jean, P., Knodlseder, J., Roques, J., Weidenspointner, G., Schanne, S., Hartmann, D., Winkler, C., \& Wunderer, C. 2006, Nature, 439, 45

Draine, B. T. 2011, Physics of the Interstellar and Intergalactic Medium (Princeton, N.J.: Princeton University Press)

Dwarkadas, V. V., Dauphas, N., Meyer, B., Boyajian, P., \& Bojazi, M. 2017, ApJ, 851, 14pp 
Fryer, C. L. 1999, ApJ, 522, 413

Fujimoto, Y., Krumholz, M. R., \& Tachibana, S. 2018, MNRAS, 480, 4025

Goriely, S. \& Arnould, M. 2001, A\&A, 379, 1113

Gounelle, M. \& Meynet, G. 2012, A\&A, 545, A4

Harper, Charles L., J. 1996, AJ, 466, 1026

Huss, G., Meyer, B. S., Srinivasan, G., Goswami, J. N., \& Sahijpal, S. 2009, Geochemica et Cosmochimica Acta, 73, 4922

Iizuka, T., Lai, Y.-J., Akram, W., Amelin, Y., \& Schonbachler, M. 2016, Earth and Planetary Science Letters, 439, 172

Jacobsen, S. 2005, in Chondrites and the Protoplanetary Disk, ed. A. Krot, E. Scott, \& B. Reipurth, Vol. 341 ASP, 548-557

Jura, M., Xu, S., \& Young, E. D. 2013, ApJ, 775, L41

Kasen, D., Metzger, B., Barnes, J., Quataert, E., \& Ramirez-Ruiz, E. 2017, Nature, 551, 80

Lugaro, M., Heger, A., Osrin, D., Goriely, S., Zuber, K., Karakas, A., Gibson, B. K., Dopherty, C. L., Lattanzio, J. C., \& Ott, U. 2014, Science, 345, 650

Martin, P., Knodlseder, J., Diehl, R., \& Meynet, G. 2009, A\&A, 506, 703

Meyer, B. S. \& Clayton, D. D. 2000, Sp.Sci.Rev., 92, 133

Rauscher, T., Heger, A., Hoffman, R., \& Woosley, S. 2002, ApJ, 576, 323

Schonbachler, M., Rehkamper, M., Halliday, A. N., Lee, D.-C., Bourot-Denise, M., Zanda, B., Hattendorf, B., \& Gunther, D. 2002, Science, 295, 1705

Smartt, S. J. 2009, Annu. Rev. Astron. Astrophys., 47, 63

Smartt, S. J., Chen, T.-W., Jerkstrand, A., et al. 2017, Nature, 551, 75

Sukhbold, T., Woosley, S. E., \& Heger, A. 2018, ApJ, 869, 22pp

Thielemann, F.-K., Eichler, M., Panov, I. V., \& Wehmeyer, B. 2017, Ann. Rev.Nuc.ES Part.Sci., 67,253

Tielens, A. 2005, in The Physics and Chemistry of the Interstellar Medium, 461-475

Tissot, F. L. H., Dauphas, N., \& Grossman, L. 2016, Science Advances, 2, 7 pp

Tsujimoto, T. \& Shigeyama, T. 2014, A\&A, 565, 4pp

Wasserburg, G. J., Busso, M., \& Gallino, R. 1996, ApJ, 466, L109

Wasserburg, G. J., Busso, M., Gallino, R., \& Nollett, K. M. 2006, Nuclear Physics A, 777, 5

Woosley, S. \& Heger, A. 2007, Phys.Rep., 442, 269

Young, E. D. 2014, Earth and Planetary Science Letters, 392, 16027

Young, E. D. 2016, ApJ, 826, 6pp

\section{Discussion}

Lissauer: Assuming Wolfe-Rayet stars are the primary source of ${ }^{26} \mathrm{Al}$ in the Solar System, what fraction of planetary systems would you expect to have similar excesses?

YounG: A consequence of discrete sampling of the stellar initial mass function is that larger star clusters more reliably produce more massive stars. Larger clusters implies formation in higher-mass star-forming regions. The fraction of planetary systems with solar-like relative concentrations of ${ }^{26} \mathrm{Al}$ should therefore correlate with the fraction of systems that form in massive star-forming regions where the high-mass end of the IMF is well sampled and WR stars are virtually guaranteed.

KRUIJSSEN: What is the nature of the molecular cloud "residency timescale" that you use to explain the radioisotope abundance as a function of their lifetime? You seem to use something like the gas depletion time (or Mgas/SFR), but cloud lifetimes are now being found to be 10-30 Myr in our recent work. How would the curve change if you use a shorter timescale?

YounG: We consider that characteristic evolutionary timescales correlate with scale, such that of order $10^{6} \mathrm{yrs}$ applies to formation of individual clusters, $\sim 3 \times 10^{7} \mathrm{yrs}$ 
applies for the longevity of giant molecular clouds, and $10^{8}$ yrs applies to the typical lifetime of molecular cloud complexes comprising spiral arms. Of these timescales, the gas depletion time is, arguably, the best measure of the time that a typical nuclide persists from its synthesis to its incorporation into a star and associated planetary system. The relative abundances of the solar-system radionuclides, especially the long-lived nuclides, are telling us that the average interval for isolation and radioactive decay was on the order of $200 \mathrm{Myr}$ leading up to the formation of the solar system. This implies that these nuclides were likely passed from cloud to cloud as a result of inefficient star formation in the clouds. Evolution curves in Figure 2 would be considerably shallower with radioactive free decay times of 10 to $30 \mathrm{Myr}$, and would not be consistent with the long-lived nuclide data irrespective of the implications for the short-lived nuclides. 